Harvard Kennedy School Misinformation Review ${ }^{1}$

December 2021, Volume 2, Issue 6

Creative Commons Attribution 4.0 International (CC BY 4.0)

Reprints and permissions: misinforeview@hks.harvard.edu

DOI: https://doi.org/10.37016/mr-2020-86

Website: misinforeview.hks.harvard.edu

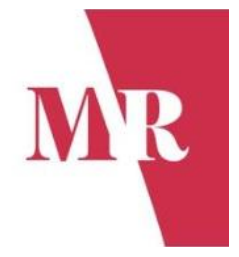

\title{
Retraction note to: Disinformation creep: ADOS and the strategic weaponization of breaking news
}

The HKS Misinformation Review retracts the article "Disinformation creep: ADOS and the strategic weaponization of breaking news" (https://doi.org/10.37016/mr-2020-52), which was published in the journal on January 18, 2021.

Authors: HKS Misinformation Review Editorial Staff

How to cite: HKS Misinformation Review Editorial Staff. (2021). Retraction note to: Disinformation creep: ADOS and the strategic weaponization of breaking news, 2(6).

Published: December 20th, 2021.

After concerns were brought to our attention by the organization that is the object of the study, challenging the validity of the findings reported by Nkonde et al., the journal commissioned an internal review, conducted by a Harvard researcher not directly affiliated with the journal. The internal review found flaws in the methodology, as well as discrepancies between the data and the findings reported by the authors, resulting in unsubstantiated conclusions drawn from their analyses. We then commissioned an external independent review to verify the findings of the initial investigation.

The external review found that Nkonde et al.'s study failed to meet professional standards of validity and reliability. The review stated that "the conclusions drawn by the authors are supported primarily by their interpretation of a few selected tweets by ADOS leadership," and that "the quantitative analysis is insufficiently connected to the conclusions" of the paper. The reviewer conducted a partial replication of the quantitative analysis and found that it did not support the authors' findings that Boseman's passing "barely registered" in the ADOS network or their conclusion that the network exhibits a "lack of concern" for COVID-19.

After the post-publication review process was completed, the authors were invited to respond to the issues identified by the two reviewers. In their response, the authors conceded several of the defects in the study identified by the internal and external reviewers. The retraction decision was not taken lightly but is one that we feel was necessary, as certain of the principal conclusions reported in this paper cannot be considered reliable or valid.

It is important to acknowledge that this outcome also represents a failure of the journal's editorial process. We, thus, intend to scrutinize our own practices, procedures, and policies to prevent similar occurrences in the future.

\footnotetext{
${ }^{1}$ A publication of the Shorenstein Center on Media, Politics and Public Policy at Harvard University, John F. Kennedy School of Government.
} 


\section{Bibliography}

Nkonde, M., Rodriguez, M. Y., Cortana, L., Mukogosi, J. K., King, S., Serrato, R., Martinez, N., Drummer, M., Lewis, A., \& Malik, M. M. (2021). Disinformation creep: ADOS and the strategic weaponization of breaking news. Harvard Kennedy School (HKS) Misinformation Review, 1(7). https://doi.org/10.37016/mr-2020-52 